\title{
Meningiomas: a comprehensive text M. Necmettin Pamir, Peter M. Black, Rudolf Fahlbusch
}

\author{
Hardcover, 773 pp, ISBN: 978-1-416056546 Saunders Elsevier
}

Nicolas de Tribolet

Published online: 8 June 2010

(C) Springer-Verlag 2010

The title of the book appropriately reflects its contents, this indeed is a comprehensive review. It contains 13 videos to illustrate certain surgical approaches.

The book is divided into 6 sections. 1) Neurobiology, 2) Diagnosis, 3) Surgery, 4) Surgery by Site of origin, 5) Radiation and Chemotherapy, 6) Special Topics.

The 64 chapters are written by different authors, experts in the field, but the presentation is remarkably homogenous. There are numerous illustrations; most of excellent quality and the text is clearly presented.

The first section includes chapters on history (particularly of the histological classification), meningeal anatomy, origin (mainly molecular genetics) Epidemiology (including hormonal influence and genetics), radiation induced meningiomas, neuropathology, biology, molecular biology and genetics (some overlap with previous chapters), brain edema and angiogenesis with its molecular mechanisms. I particularly liked the chapter on meningeal anatomy. The detailed description of the vascular supply to the meninges is of utmost importance in the surgery of these tumours.

The second section addresses the clinical presentation with a particularly good chapter on Neuroophtahlmology followed by chapters on CT, MRI, MRI and PET, Angiography and a short chapter on automatic tumor growth detection.

The third section deals with general aspects of surgery such as decision making, perioperative management, anaes- thesia and intensive care, neurophysiologic monitoring and considerations on the cerebral venous system.

The fourth section addresses surgical technique by site of origin of the meningiomas. All chapters are written by well recognized neurosurgeons and deal with every possible location. For petroclival meningiomas for example, the posterior petrosal, the anterior transpetrosal, the retrosigmoid and the presigmoid keyhole approaches are described. A special chapter is dedicated to pediatric meningiomas. As the editors mention in the preface each author shares his personal experience.

The fifth section deals with the various radiation therapy techniques, fractionated, Gammaknife, radiosurgery (overlaps with Gammaknife), LINAC, proton beam and cyberknife. The chapter on chemotherapy includes hormonal therapy. The last chapter opens perspectives for gene therapy.

The sixth and last section contains chapters addressing recurrence, anaplastic meningiomas, meningioma metastasis, the patient's view, emerging surgical techniques, experimental models and finally future research.

The only thing that is missing in this book is a chapter on embolization, this is rather surprising for a review that wants to be comprehensive. Still this is an outstanding volume which is of interest not only to neurosurgeons but also to neuroradiologists, neurologists and neurooncologists.
N. de Tribolet $(\bowtie)$

Neurochirurgie, HUG,

1211 Geneva, Switzerland

e-mail: Nicolas.DeTribolet@unige.ch 